\title{
COMPORTAMENTO TÉRMICO DO 8-QUINOLINOL E SEUS NITRO-DERIVADOS
}

\author{
Henrique E. Zorel Jr.", Ademir G. C. Costalonga, Marisa S. Crespi e Clóvis A. Ribeiro
}

Instituto de Química, Universidade Estadual Paulista Júlio de Mesquita Filho, CP 355, 14801-970 Araraquara - SP

Recebido em 31/5/00; aceito em 15/3/01

THERMAL BEHAVIOR OF THE 8-QUINOLINOL AND THEIR NITRO-DERIVATIVES. The compounds 5-nitro-8-quinolinol and 5,7-dinitro-8-quinolinol were obtained by nitration of the chelant 8-quinolinol. The compounds were characterized through EA, MNR, XRD, IR, TG, DTA and DSC. It was verified through thermal analysis that the compounds show consecutive processes of sublimation, fusion and vaporization. During the vaporization process, partial thermal decomposition was observed, with formation of carbonaceous residues. Considering a slower heating rate, the sublimation is the prevalent process to the nitro-derivatives while the vaporization is the main process to 8-quinolinol. The thermal stability follows the decreasing order from 5,7-dinitro-8-quinolinol to 5-nitro-8-quinolinol to 8-quinolinol.

Keywords: thermal behavior; 8-quinolinol; nitro-derivatives.

\section{INTRODUÇÃO}

O quelante 8-quinolinol é objeto de estudos em diferentes sistemas, como na separação e quantificação de metais, onde a formação dos complexos metálicos sólidos é altamente dependente do $\mathrm{pH}$ e meio de precipitação ${ }^{1,2}$. Avaliação destes complexos quanto à estrutura, estequiometria e comportamento térmico encontram-se relatados ${ }^{3-9}$. A influência de substituintes, como os halogêneos, grupos carboxila e grupos acetila, na estrutura do quelante, conduz à variações nas condições de preparação e no comportamento térmico dos complexos sólidos, devido à modificações na distribuição eletrônica nos anéis do quelante ${ }^{10,11}$, conduzindo à mudanças de estabilidade térmica e composição dos resíduos ${ }^{12-14}$. Os estudos do 8-quinolinol e seus derivados em sistemas biológicos ocorrem em função de interações com estruturas presentes no organismo ${ }^{15-23}$. A. Y. Shen et al. ${ }^{24}$ investigaram a utilização do 8-quinolinol, como bases de Mannich, no combate ao câncer em células humanas enquanto que M. P. Zawistoski ${ }^{25}$ estudou a utilização de 5nitro-8-quinolinol como um grupo de proteção para enxofre na síntese de tioquinolinas 5-carboxi-8-substituídas, utilizadas como intermediários para a síntese de inibidores $\mathrm{H}^{+}, \mathrm{K}^{+}$-ATPase. A adição do grupo $\mathrm{NO}_{2}$ nas posições 5 e/ou 7 na estrutura do 8quinolinol, resulta em produtos utilizados como fungicida e bactericida ${ }^{26,27}$. M. A. Zayed et $\mathrm{al}^{28}$ investigaram o comportamento térmico do quelante 5,7-dinitro-8-quinolinol e seu complexo com urânio por espectrometria de massa e análise térmica diferencial apresentando resultados, em relação ao quelante, diferentes daqueles reportados na literatura ${ }^{29}$ e por nós obtidos.

Devido às diferentes aplicações que o quelante 8-quinolinol e seus nitro-derivados podem apresentar, faz-se necessário uma correta caracterização destes compostos bem como determinar a influência da temperatura em seu comportamento. Neste trabalho, a preparação e caracterização dos nitro-derivados do 8 quinolinol são apresentadas, juntamente com um estudo comparativo destes compostos quanto ao comportamento térmico em diferentes condições de análise.

\section{PARTE EXPERIMENTAL}

Solução aquosa de nitrito de sódio foi adicionada lentamente

*e-mail: henrzore@posgrad.iq.unesp.br a uma solução aquosa de 8-quinolinol (I), recristalizada em acetona, em meio de ácido clorídrico concentrado e gelo, sob rigorosa agitação por 1 hora à temperatura na faixa de 0 a $4{ }^{\circ} \mathrm{C}$. A mistura foi mantida em repouso a $0{ }^{\circ} \mathrm{C}$ por cinco horas e o intermediário 5-oxima-8-cetoquinolina (II) foi filtrado e lavado com água destilada fria, seco a $60{ }^{\circ} \mathrm{C}$ em estufa e recristalizado em acetona. Adicionou-se a este intermediário (II) solução aquosa de ácido nítrico e após agitação ocasional por 1 hora e 15 minutos, a mistura foi diluída com água destilada, resfriada a $0{ }^{\circ} \mathrm{C}$ e alcalinizada com solução de hidróxido de potássio fria. O sal de potássio foi decomposto com ácido acético glacial e o produto final, 5-nitro-8-quinolinol (III), foi filtrado e lavado com água destilada fria, seco a $60{ }^{\circ} \mathrm{C}$ e recristalizado em acetona. $\mathrm{O}$ composto 5,7-dinitro-8-quinolinol (IV) foi obtido pela adição de excesso de ácido nítrico durante a preparação da 5-nitro-8quinolinol, seguido de aquecimento, resultando num precipitado o qual foi lavado com água destilada, seco a $60{ }^{\circ} \mathrm{C}$ em estufa e recristalizado em acetona.

As estruturas dos compostos estudados estão apresentadas abaixo, segundo o processo de nitração utilizado:<smiles>Oc1cccc2cccnc12</smiles><smiles>O=C1C=CC(=O)c2ccccc21</smiles>

II<smiles>O=[N+]([O-])c1ccc(O)c2ncccc12</smiles>

III<smiles>O=[N+]([O-])c1cc([N+](=O)[O-])c2cccnc2c1O</smiles>

IV
A caracterização dos compostos foi realizada por análise elementar, utilizando equipamento da CE Instruments, modelo EA 1110 CHNS-O. A substituição no anel foi verificada por ressonância magnética nuclear do próton ${ }^{1} \mathrm{H}$, com Espectrofotômetro BRUKER AC 200F, sendo as soluções para análise obtidas utilizando-se acetona deuterada como solvente. Difratometria de raios $\mathrm{X}$, método do pó, foi realizada com Difratômetro Horizontal HGZ/4B em montagem Bragg-Bretano, com radiação de cobalto $(\lambda=1.78897 \mathrm{~nm})$ em exposição até $60^{\circ}(2 \theta)$. Espectroscopia de absorção na região do infravermelho foi realizada por Espectrofotômetro de Infravermelho Nicolet, modelo FT Impact 400, com as amostras sendo preparadas em pastilhas de brometo de potássio. $\mathrm{O}$ comportamento 
térmico foi estudado por termogravimetria/análise térmica diferencial, utilizando equipamento SDT 2960, da TA Instruments, sob atmosfera dinâmica de nitrogênio $(100 \mathrm{~mL}$ $\min ^{-1}$ ) e diferentes razões de aquecimento. A pureza dos compostos foi verificada por calorimetria exploratória diferencial, equipamento DSC 2910, da TA Instruments, sob atmosfera dinâmica de nitrogênio $\left(100 \mathrm{~mL} \mathrm{~min}{ }^{-1}\right)$ e razão de aquecimento de $2,5^{\circ} \mathrm{Cmin}^{-1}$, utilizando massa de amostra em torno de 2 mg, em cadinho de alumínio hermeticamente fechado.

\section{RESULTADOS E DISCUSSÃO}

Todos os compostos foram obtidos na forma de monocristal sendo as estruturas dos nitro-derivados determinadas por difratometria de raios $\mathrm{X}$ do monocristal e em fase final de refinamento dos dados, com exceção do intermediário 5-oxima8-cetoquinolina, inédito na literatura, o qual já está com seus dados estruturais determinados e submetidos ao banco de dados do Cambridge Crystallographic Data Centre ${ }^{30}$. O composto acima citado apresenta estrutura diferente ao relatado na literatura $^{31,32}$, cujos autores indicam a formação do intermediário 5-nitroso-8-quinolinol. A Tabela 1 contém os dados obtidos por análise elementar para determinação da estequiometria dos compostos.

Os valores obtidos estão próximos àqueles esperados, confirmando a estequiometria dos compostos $8 \mathrm{Q}\left(\mathrm{C}_{9} \mathrm{H}_{7} \mathrm{NO}\right)$, 5-nitro$8 \mathrm{Q}\left(\mathrm{C}_{9} \mathrm{H}_{6} \mathrm{~N}_{2} \mathrm{O}_{3}\right)$ e 5,7-dinitro-8Q $\left(\mathrm{C}_{9} \mathrm{H}_{5} \mathrm{~N}_{3} \mathrm{O}_{5}\right)$. No entanto, a análise elementar não fornece informações à respeito da posição no anel em que ocorreu a substituição. Para tanto, realizou-se análise por ressonância magnética nuclear do próton ${ }^{1} \mathrm{H}$.

$\mathrm{O}$ espectro de ressonância do 8-quinolinol apresenta sinais na forma de duplete para os prótons ligados aos carbonos 1,3 e $7(\delta=8,80 ; 8,34 ; 7,15)$. Para os prótons ligados aos carbonos 2 e $6(\delta=7,58 ; 7,39)$ os sinais apresentam-se na forma de duplo duplete. O próton do carbono 5 pode estar acoplado ao próton do carbono na posição 7 , estando o sinal na mesma região indicada para os prótons nas posições 2 e 6 . O composto 5-nitro-8-quinolinol apresenta em seu espectro quatro sinais na forma de duplete, atribuídos aos prótons ligados aos carbonos $1,3,6$ e $7(\delta=9,27 ; 9,00 ; 8,62 ; 7,30)$. Um sinal na forma de duplo duplete é atribuído ao próton do carbono $2(\delta=7,91)$, o qual está acoplado aos prótons das posições 1 e 3 . A não observação de um sinal na posição 5 indica que a substituição ocorreu nesta posição, confirmando a obtenção do composto. Os resultados apresentados estão em concordância com a literatura $^{33}$. Não foi possível caracterizar o composto 5,7-dinitro8-quinolinol por ressonância magnética nuclear devido à baixa solubilidade em acetona, dimetilsulfóxido e clorofórmio deuterados. Entretanto a análise por difratometria de raios $\mathrm{X}$ do monocristal confirmou as substituições nas posições desejadas, com o composto apresentando estrutura monoclínica ${ }^{34}$.

O espectro de absorção na região do infravermelho do 8quinolinol apresenta uma banda de absorção em torno de 3100 $\mathrm{cm}^{-1}$, de baixa intensidade e larga, atribuída ao estiramento da ligação $\mathrm{O}-\mathrm{H}$ e apresenta-se desta forma devido à formação de ligações de hidrogênio intramolecular no quelante, as quais envolvem o grupo hidroxila e o átomo de nitrogênio. Observou-se bandas de absorção nas regiões de $1571 \mathrm{e} 1504 \mathrm{~cm}^{-1}$, atribuídas aos estiramentos $\mathrm{C}=\mathrm{C}$ e $\mathrm{C}=\mathrm{N}$ do anel. Uma banda intensa atribuída à deformação no plano $\mathrm{O}-\mathrm{H}$, acoplada ao estiramento C-O, foi observada em $1379 \mathrm{~cm}^{-1}$. Uma banda em $1278 \mathrm{~cm}^{-1}$ foi atribuída à deformação no plano de $\mathrm{O}-\mathrm{H}$. Em $1207 \mathrm{~cm}^{-1}$ observou-se uma banda de aborção atribuída à deformação axial de C-O e à deformação no plano de C-H. Uma banda em $1091 \mathrm{~cm}^{-1}$ é atribuída ao estiramento C-O. Bandas observadas na região de 779 e $632 \mathrm{~cm}^{-1}$ foram atribuídas à deformação angular fora do plano.

O espectro de absorção na região do infravermelho do composto 5-nitro-8-quinolinol apresentou bandas de absorção de média intensidade na região de 1200 a $1000 \mathrm{~cm}^{-1}$ e de forte intensidade na região de 1600 a $1300 \mathrm{~cm}^{-1}$, características de compostos nitro-aromáticos ${ }^{35}$. Embora sejam observadas bandas nestas regiões do espectro, torna-se difícil distingui-las das bandas observadas nos espectros de absorção do quelante 8quinolinol, por ocorrerem na mesma região. Desta forma, as bandas que caracterizaram o composto 5-nitro-8-quinolinol foram observadas nas regiões de 1418 e $1318 \mathrm{~cm}^{-1}$, bem como aquelas em 793 e $838 \mathrm{~cm}^{-1}$, atribuídas ao estiramento $\mathrm{N}-\mathrm{O}^{35}$. Uma banda de absorção na região de $2926 \mathrm{~cm}^{-1}$ também é característica da substituição no anel aromático por grupos NO e $\mathrm{NO}_{2}$. O composto 5,7-dinitro-8-quinolinol apresentou bandas de absorção nas regiões do espectro entre 1600 e $1400 \mathrm{~cm}^{-1}$, características de substituição orto e para ${ }^{35}$. Apresentou também uma banda em $2914 \mathrm{~cm}^{-1}$, característica dos grupos $\mathrm{NO}$ e $\mathrm{NO}_{2}$, como mencionado anteriormente.

Os difratogramas de raios $\mathrm{X}$ destes compostos apresentaram picos intensos em regiões distintas, indicando alta cristalinidade e ausência de isomorfismo.

Os compostos foram caracterizados pelas curvas DSC, Figura 1, para determinação do ponto de fusão, cujo pico foi utilizado para determinação da pureza.

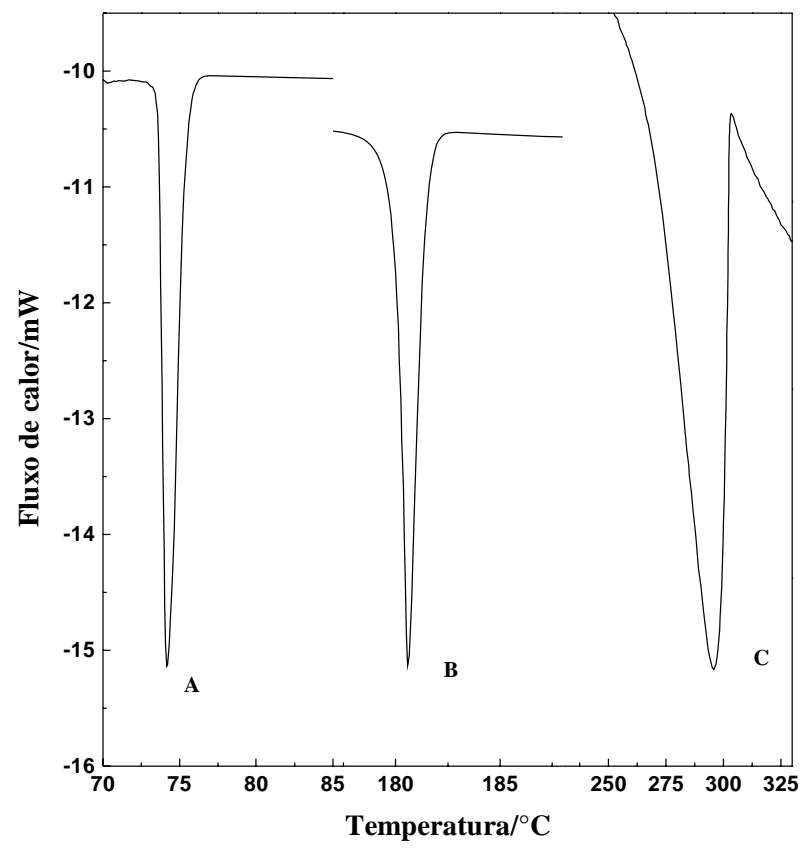

Figura 1. Curvas DSC dos compostos 8-quinolinol (A), 5-nitro-8-quinolinol (B) e 5,7-dinitro-8-quinolinol (C), obtidas em atmosfera dinâmica de nitrogênio (100 $\mathrm{mL} \mathrm{min}^{-1}$ ) e razão de aquecimento de $2,5{ }^{\circ} \mathrm{C} \mathrm{min}^{-1}$.

Tabela 1. Valores obtidos por análise elementar do 8-quinolinol e seus nitro-derivados.

\begin{tabular}{lccccc}
\hline Compostos & $M / \mathrm{g} \mathrm{mol}^{-1}$ & $\mathrm{~N} / \%$ & $\mathrm{C} / \%$ & $\mathrm{H} / \%$ & $\mathrm{Cor}$ \\
\hline 8-quinolinol & 145,08 & $9,57(9,65)$ & $74,39(74,47)$ & $4,95(4,86)$ & Branco \\
5-nitro-8-quinolinol & 190,07 & $14,86(14,73)$ & $56,76(56,85)$ & $3,08(3,18)$ & Amarelo \\
5,7-dinitro-8-quinolinol & 235,07 & $17,16(17,57)$ & $46,32(45,97)$ & $2,18(2,14)$ & Marrom \\
\hline
\end{tabular}

Obs. Os valores esperados estão entre parênteses. 
Observa-se que os compostos 8-quinolinol e 5-nitro-8quinolinol apresentam pico endotérmico agudo, característico da fusão, o mesmo não ocorrendo com o composto 5,7-dinitro8-quinolinol, o qual apresenta formato mais largo e baixa definição da linha base. O alargamento do pico foi atribuído à decomposição parcial do composto, próximo à fusão, que será discutido nas curvas TG/DTA. Em função deste fato, os cálculos de $\Delta \mathrm{H}_{\text {fusão }}$ e pureza, para o composto 5,7-dinitro-8quinolinol, não foram realizados.

Os resultados referentes à pureza, observados na Tabela 2, foram obtidos com base nas equações de Van't $\operatorname{Hoff}^{36}$, pela análise dos picos de fusão. Pode-se observar que os compostos apresentam alto grau de pureza, indicando a eficiência da recristalização em meio de acetona, e que as temperaturas de fusão estão em acordo com a literatura ${ }^{37}$.

O comportamento térmico dos compostos foi estudado por TG/DTA, em atmosfera dinâmica de nitrogênio $\left(100 \mathrm{~mL} \mathrm{~min}{ }^{-1}\right)$, sendo as curvas apresentadas na Figura 2.

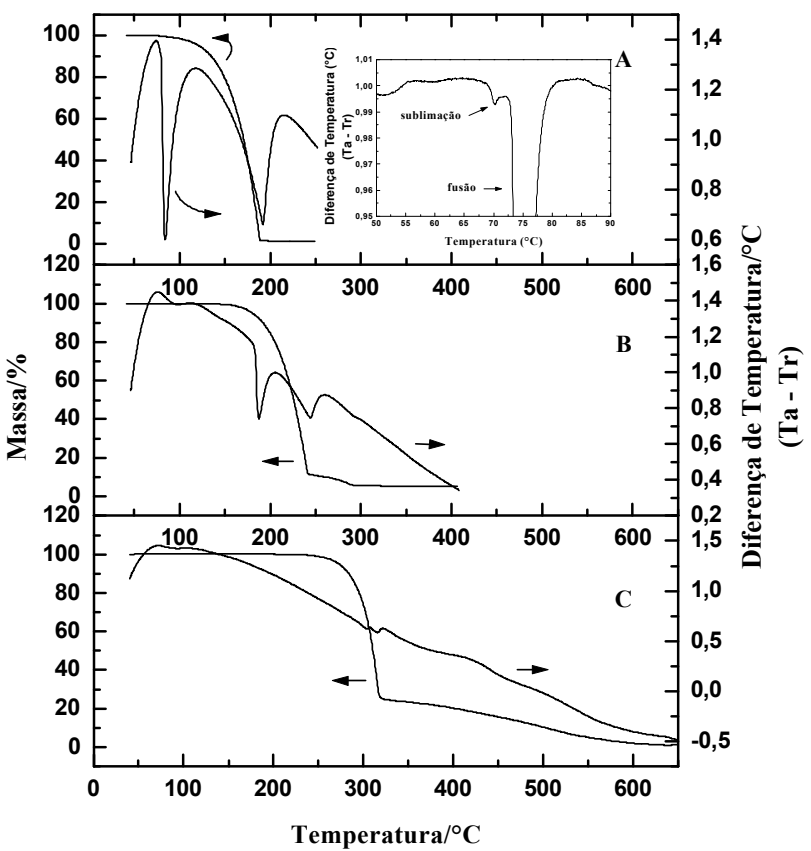

Figura 2. Curvas TG/DTA dos compostos 8-quinolinol (A), 5-nitro8-quinolinol $(B)$ e 5,7-dinitro-8-quinolinol (C), obtidas em atmosfera

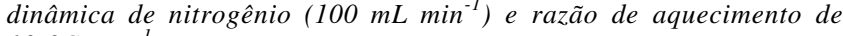
$10{ }^{\circ} \mathrm{C} \mathrm{min}^{-1}$.

A curva TG do 8-quinolinol, Figura 2A, apresenta uma única etapa de perda de massa, atribuída aos processos de sublimação/vaporização do composto. O processo de sublimação, indicado por um pequeno pico endotérmico na curva DTA (destaque) é evidenciado pela perda de massa antes que ocorra a fusão do composto, a qual também está indicada pelo pico endotérmico em $73{ }^{\circ} \mathrm{C}$ na curva DTA. Um terceiro pico endotérmico, com máximo em $188^{\circ} \mathrm{C}$, é atribuído ao processo de vaporização do material fundido.

Observa-se que a curva TG do composto 5-nitro-8-quinolinol, Figura 2B, apresenta duas etapas de perda de massa. Na primeira etapa, o composto apresenta o mesmo comportamento que a 8quinolinol, com sublimação seguida da vaporização do material fundido. Embora não se observe o pico endotérmico referente à sublimação, esta é evidenciada pela perda de massa antes da fusão, a qual ocorre em $180{ }^{\circ} \mathrm{C}$, indicada pelo pico endotérmico na curva DTA. Estes eventos podem ser melhor observados na Figura 3. Um segundo pico endotérmico é observado na curva DTA com máximo em $240{ }^{\circ} \mathrm{C}$, e é atribuído à vaporização, como mencionado anteriormente. A segunda etapa de perda de massa é atribuída à eliminação do resíduo obtido pela decomposição do composto, antes que este tenha sido totalmente vaporizado. O resíduo remanescente é eliminado lentamente, devido a utilização de atmosfera não oxidante.

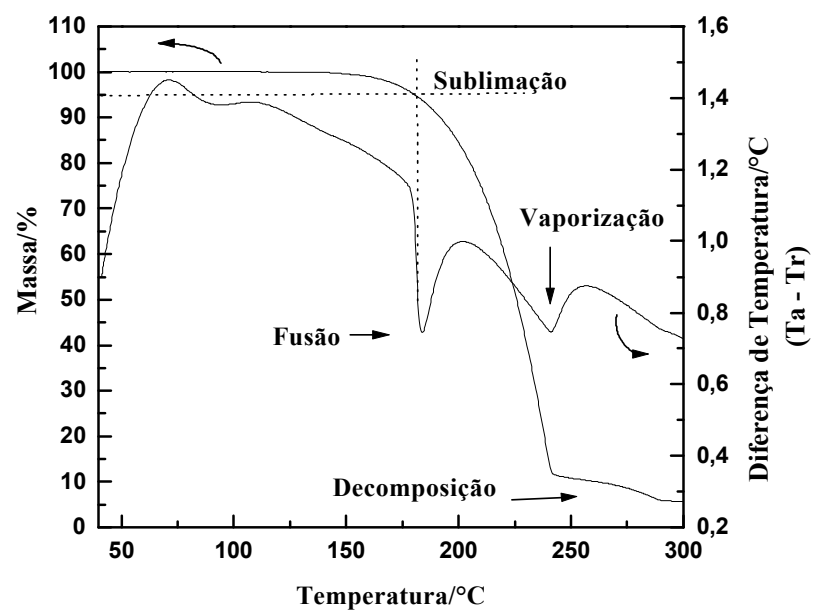

Figura 3. Curvas TG/DTA do composto 5-nitro-8-quinolinol obtidas

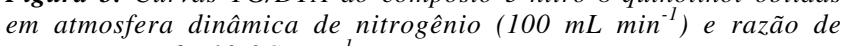
aquecimento de $10{ }^{\circ} \mathrm{C} \mathrm{min}^{-1}$.

O composto 5,7-dinitro-8-quinolinol, Figura 2C, apresenta o mesmo comportamento térmico que o 5-nitro-8-quinolinol. A primeira etapa de perda de massa é atribuída aos processos de sublimação e vaporização. O processo de vaporização é indicado por pico endotérmico na curva DTA, com máximo em $316{ }^{\circ} \mathrm{C}$, posterior ao pico de fusão em $302{ }^{\circ} \mathrm{C}$. A segunda etapa, assim como para o 5-nitro-8-quinolinol, é atribuída à eliminação de resíduo, entre 320 e $700{ }^{\circ} \mathrm{C}$, resultante da decomposição do composto antes de sua total vaporização.

Neste ponto observa-se a influência da razão de aquecimento sobre o pico de fusão deste composto, devido a decomposição. Na curva DSC, obtida em razão de aquecimento de $2,5^{\circ} \mathrm{C}$ $\mathrm{min}^{-1}$, observou-se a fusão em $266{ }^{\circ} \mathrm{C}$, enquanto que na curva DTA, obtida a $10{ }^{\circ} \mathrm{C} \min ^{-1}$, a temperatura de fusão observada foi de $302{ }^{\circ} \mathrm{C}$. Este fato é atribuído à influência da decomposição do composto, sobre o pico de fusão obtido na curva DSC, o qual se apresentou mais largo que os demais compostos, justificando a não utilização deste pico para cálculos de $\Delta \mathrm{H}_{\text {fusão }}$ e pureza. Embora o composto 5-nitro-8-quinolinol também sofra decomposição, pode-se observar que esta não influencia na fusão, pela simples comparação das temperaturas de fusão e de decomposição, indicada pelo início da segunda etapa de perda de massa na curva TG, para os dois compostos nitro-

Tabela 2. Valores obtidos para análise de pureza por DSC do 8-quinolinol e 5-nitro-8-quinolinol.

\begin{tabular}{|c|c|c|c|c|}
\hline \multirow[t]{2}{*}{ Compostos } & \multicolumn{2}{|c|}{$\mathrm{T}_{\text {fusão }} /{ }^{\circ} \mathrm{C}$} & \multirow{2}{*}{$\begin{array}{l}\Delta \mathrm{H}_{\text {fusão }} \\
\mathrm{kJ} \mathrm{mol}^{-1}\end{array}$} & \multirow{2}{*}{$\begin{array}{c}\text { Pureza } \\
\%\end{array}$} \\
\hline & literatura & observado & & \\
\hline 8-quinolinol & 76 & 73,62 & 22,1 & 98,6 \\
\hline 5-nitro-8-quinolinol & 180 & 180,04 & 24,7 & 99,9 \\
\hline 5,7-dinitro-8-quinolinol & $276 ; 320$ & 266,14 & - & - \\
\hline
\end{tabular}


Tabela 3. Percentagem de material sublimado observado nas curvas TG, com base nas temperaturas de fusão dos compostos, obtidas sob diferentes razões de aquecimento.

\begin{tabular}{lccccc}
\hline Compostos & \multicolumn{5}{c}{ Razão de aquecimento/ ${ }^{\circ} \mathrm{C}$ min $^{-1}$} \\
\hline & 1 & 2.5 & 5 & 10 & 20 \\
\hline 8-quinolinol & 2,20 & 1,21 & 0,51 & 0,23 & 0,14 \\
5-nitro-8-quinolinol & 54,73 & 19,76 & 11,27 & 4,95 & 2,31 \\
5,7-dinitro-8-quinolinol & 28,52 & 14,65 & 6,40 & 3,21 & 1,49 \\
\hline
\end{tabular}

derivados. O fato do composto 5,7-dinitro-8-quinolinol apresentar maior quantidade de resíduo é atribuído justamente à proximidade das temperaturas de fusão e decomposição o que conduz também à uma menor definição dos picos de fusão e vaporização observados nas curvas DTA.

Esta aproximação dos eventos térmicos deve ter conduzido à indicação equivocada por F. Bedall e E. Shmitt ${ }^{29}$, que apresentam duas temperaturas de fusão para o composto 5,7-dinitro8-quinolinol (Tabela 2). Ainda para este composto, M. A. Zayed et al. ${ }^{28}$ não observaram fusão na curva DTA, apresentando apenas picos exotérmicos, os quais foram atribuídos à decomposição do composto em $150{ }^{\circ} \mathrm{C}$, bastante inferior àquela por nós apresentada na curva TG.

Observa-se nas curvas TG que aumentando-se a quantidade de grupo $\mathrm{NO}_{2}$ na estrutura, aumenta-se a estabilidade térmica do composto. Isto se dá pela mudança da distribuição eletrônica no anel do grupo fenol ${ }^{13}$.

Os compostos foram analisados por TG em diferentes razões de aquecimento, verificando-se que os processos descritos na Figura 2 não são alterados. Desta forma, escolheu-se o composto 8-quinolinol como representativo, sendo as curvas apresentadas na Figura 4.

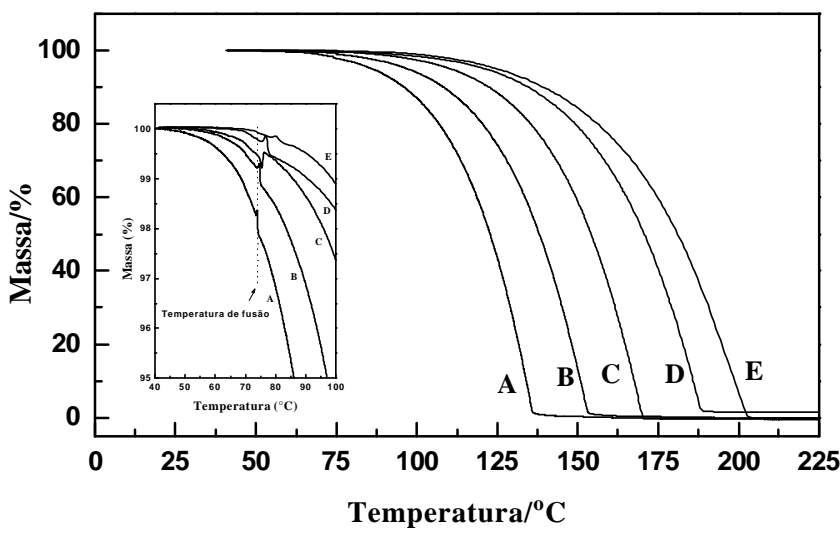

Figura 4. Curvas TG do composto 8-quinolinol obtidas em atmosfera dinâmica de nitrogênio $\left(100 \mathrm{~mL} \mathrm{~min}^{-1}\right)$ e razões de aquecimento de $1 ; 2,5 ; 5 ; 10$ e $20{ }^{\circ} \mathrm{C} \mathrm{min}^{-1}$.

Observa-se que as temperaturas inicial e final de perda de massa são maiores quanto maior a razão de aquecimento. Este fato está relacionado à cinética do processo que, no caso, é a sublimação, por onde inicia-se a etapa de perda de massa dos compostos. Se a sublimação ocorresse de forma muito rápida, menor seria a diferença de temperatura observada para as diferentes razões de aquecimento. Um estudo cinético destes compostos está sendo reportado ${ }^{38}$.

Uma vez que a cinética de sublimação não foi alterada pela variação da razão de aquecimento, a quantidade de material sublimado, como pode ser visto no destaque da Figura 4, é menor quanto maior a razão de aquecimento, devido à aproximação mais rápida da temperatura de fusão dos compostos. A Tabela 3, contém os valores da quantidade de material sublimado em função da razão de aquecimento para os compostos.
Estes resultados indicam que a cinética de sublimação destes compostos é diferente, obedecendo a seguinte ordem crescente de quantidade de material sublimado: 8-quinolinol $<5,7$ dinitro-8-quinolinol $<$ 5-nitro-8-quinolinol.

\section{CONCLUSÕES}

Neste trabalho apresentou-se a preparação e caracterização dos compostos 5-nitro-8-quinolinol e 5,7-dinitro-8-quinolinol a partir do 8-quinolinol. Verificou-se que os compostos foram obtidos na forma desejada, apresentando alto grau de pureza e cristalinidade.

Observou-se que o comportamento térmico dos compostos passa por processos de sublimação, fusão, vaporização, e que os nitroderivados apresentam decomposição. Considerando-se baixa razão de aquecimento, a perda de material por sublimação torna-se predominante no composto 5-nitro-8-quinolinol enquanto que para os compostos 8-quinolinol e 5,7-dinitro8quinolinol predomina o processo de vaporização.

\section{AGRADECIMENTOS}

Os autores agradecem ao CNPq/PADCT-II (processo 62.0651/94.6) e a FAPESP (processo 97/05613-6) pelo suporte financeiro.

\section{REFERÊNCIAS}

1. Ohlweiler. O. A.; Química Analítica Quantitativa, $2^{\text {nd }} \mathrm{Ed}$, v 1, Livros Técnicos e Científicos, Rio de Janeiro, 1978; p 312 .

2. Vogel, A. I.; A Textbook of Quantitative Inorganic Analysis, $3^{\text {th }}$ Ed, ELBS and Longman, London, 1978; p $128 ; 386$

3. Zorel Jr., H. E.; Ribeiro, C. A.; Crespi, M. S.; Anais Assoc. Bras. Quím. 1998, 47, 377.

4. Dubey, B. L.; Tiwari, N.; J. Therm. Anal., 1997, 48, 885.

5. Zorel Jr., H. E.; Guinesi, L. S.; Ribeiro, C. A.; Crespi. M. S.; Mater. Lett. 2000, 42, 16.

6. Murthy, Y. S. N.; Gopolan, R.; Raoot, S.; Rajasekharan, T.; Ravi, S.; Bai, V. S.; Mater. Lett. 1990, 9, 154.

7. Kokkonen, P.; Palmu, L.; Lajunen, L. H. J.; Thermochim. Acta 1987, 114, 329.

8. Guinesi, L. S.; Zorel Jr., H. E.; Ribeiro, C. A.; Crespi, M. S.; Anais Assoc. Bras. Quím. 1999, 48, 221.

9. Crespi, M. S.; Ribeiro, C. A.; Greenhalf, V. C. M.; Zorel Jr., H. E.; Quim. Nova 1999, 22, 41.

10. Steger, H. F.; Corsini, A.; J. Inorg. Nucl. Chem. 1973 $35,1621$.

11. Steger, H. F.; Corsini, A.; J. Inorg. Nucl. Chem. 1973 , $35,1637$.

12. Hollingshead, R. G. N.; Anal. Chim. Acta 1955, 12, 401.

13. Hollingshead, R. G. N.; Anal. Chim. Acta 1955, 12, 201.

14. Guerreiro, C. T. R.; Ribeiro, C. A.; Crespi, M. S.; Torres, C.; J. Therm. Anal. Calorim. 1999, 56, 519.

15. Zajoncova, L.; Frebort, I.; Luhova, L.; Sebela, M.; Galuszka, P.; Pec, P.; Biochem. Mol. Biol. Int. 1999, 47, 47.

16. Narayanan, R.; LeDuc, B.; J. Pharmaceut. Biomed. 2000, $22,527$. 
17. Giraudi, G.; Baggiani, C.; Giovannoli, C.; Marletto, C.; Vanni, A.; Anal. Chim. Acta 1999, 378, 225.

18. Vazquez, B.; Ginebra, M. P.: Gil, F. J.; Planell, J. A.; Bravo, A. L.; SanRoman, J.; Biomaterials 1999, 20, 2047.

19. Hall, H. A.; Martoran, P.; Toxicol Appl. Pharm. 1966, $8,343$.

20. Sharma, Y.; Shivahare, G. C.; J. Chin. Chem. Soc-Taip. 1980, 27, 129 .

21. Gupta, R. D.; Manku, G. S.; Bhat, A. N.; Jain, B. D.; Anal. Chim. Acta 1970, 50, 109.

22. Kuzyakova, N. Y.; Golovina, A. P.; J. Anal. Chem$U S S R+1983,38,773$.

23. Da Silva, M. A. V.; Monte, M. J. S.; Matos, M. A. R.; J. Chem. Thermodyn. 1989, 21, 159.

24. Shen, A. Y.; Wu, S. N.; Chiu, C. T.; J. Pharma. Pharmacol. 1999, 51, 543.

25. Zawistoski, M. P.; J. Heterocycl. Chem. 1991, $28,657$.

26. Desvignes, A.; Leguem, P.; Ann. Pharm. Franc. 1963, $21,803$.

27. Medic-Saric, M.; Maysinger, D.; Movrin, M.; Dvorzak, I.; Chemotherapy 1980, 26, 263.

28. Zayed, M. A.; ElDien, F. A. N.; AbdElGany, A. R.; Gyoryova, K.; J. Therm. Anal. 1997, 50, 487.

29. Harris, G.; Dictionary of Organic Compounds, $4^{\text {th }}$ Ed, v
3, Eyre \& Spottiswoode Publishers Ltd, London, 1965; p 1683.

30. Zorel Jr., H. E.; Costalonga, A. G. C.; Ellena, J.; Crespi, M. S.; Ribeiro, C. A.; Cambridge Crystallographic Data Centre, 2000, CCDC number 144266.

31. Gershon, H.; McNeil, M. W.; J. Heterocycl. Chem. 1971, 8,821 .

32. Voronin, V. G.; Petrova, I. D.; Leksin, A. N.; Shermer, Y.; Ankin, B. V.; Khim. Farm. Zh+ 1976, 10, 82.

33. Pouchert, C. J.; The Aldrich Library of NMR Spectra, $2^{\text {nd }}$ Ed, v 2, Aldrich Chemical Company, Inc., Molwaukee, $1983 ;$ p 743 D.

34. Ellena, J.; Zorel Jr., H. E.; Costalonga, A. G. C.; Crespi, M. S.; Ribeiro, C. A.; Act Crystallographic C, 2000, submetido.

35. Socrates, G.; Infrared characteristic group frequences tables and charts, $2^{\text {nd }}$ Ed, John Wiley \& Sons, Chichester, 1994; p 148-150.

36. Hatakeyama, T.; Quinn, F. X.; Thermal Analysis: Fundamentals and Applications to Polymer Science. John Wiley \& Sons, Chichester, 1994; p 89-92.

37. Budavari, S. In The Merck Index, Merck \& Co., Inc., $12^{\text {th }}$ Ed, New Jersey, 1996; p 4890; 6752.

38. Crespi, M. S.; Zorel Jr., H. E.; Ribeiro, C. A.; Costalonga, A. G. C.; Torres, C.; J. Therm. Anal. Calorim. 2001, 64, 783. 